\title{
IN SILICO MLST, SCCMEC AND SPA TYPING OF HUMAN MRSA STRAINS AND DETERMINATION OF ANTIMICROBIAL RESISTANCE GENES
}

\author{
Mehmet Demirci ${ }^{1}$, Akin Yigin $^{2}$, Seda Ekici ${ }^{3}$ \\ ${ }^{1}$ Kirklareli University, Faculty of Medicine, Department of Medical Microbiology, Kirklareli, TURKEY \\ ${ }^{2}$ Harran University, Faculty of Veterinary Medicine, Department of Genetics, Sanliurfa, TURKEY \\ ${ }^{3}$ Veterinary Control Central Research Institute, Ankara, TURKEY
}

Address for Correspondence: Seda EKICI, E-mail: seda.ergen@hotmail.com

Received: 16.07.2021; Accepted: 16.08.2021; Available Online Date: 20.09.2021

(C) Copyright 2021 by Dokuz Eylül University, Institute of Health Sciences - Available online at https://dergipark.org.tr/en/pub/jbachs

Cite this article as: Demirci M, Yigin A, Ekici S. In Silico MLST, SCCmec and spa Typing of human MRSA Strains and Determination of Antimicrobial Resistance Genes. J Basic Clin Health Sci 2021; 3: 171- 178.

\begin{abstract}
Objective: The incidence of MRSA still remains an important public health problem. The aim of study was to perform in silico analysis of MLST, SCCmec, spa type, evolutionary similarity and whole-genome sequencing (WGS) based antimicrobial susceptibility testing by using genomic data of MRSA strains isolated from human infections in different countries.

Methods: WGS data of 30 MRSA strains were obtained as etiological agents were download from NCBI. Phylogeny analysis with large data were performed via CSI Phylogeny online software. SCCmec, MLST and spa typing were performed using the software at the Center for Genomic Epidemiology. ResFinder 4.0 was used to perform WGS based antimicrobial susceptibility testing.

Results: After in silico analysis of 30 MRSA strains, 14 different spa types, 11 different sequence types, and 9 different SCCmec types were detected. T037, ST239, and SCCmec_type_III(3A) were the most detected spa, MLST, and SCCmec types respectively. WGS based antimicrobial susceptibility testing results were analyzed, 28, 27, and 26 out of 30 MRSA strains carried aminoglycoside tetracycline and fluoroquinolone resistance genes respectively.

Conclusions: According to our in silico analysis results, we found that similar typing profiles could be observed in the strains in different geographical locations and certain types of spa, MLST, and SCCmec can coexist.
\end{abstract}

Keywords: MRSA, MLST, SCCmec, in silico analysis, WGS based antimicrobial test

\section{INTRODUCTION}

Methicillin-resistant Staphylococcus aureus (MRSA) is an important health problem worldwide due to its multi-drug resistance ability (1). MRSA has been one of the leading pathogens since it was identified in 1960 and is recognized as one of the primary pathogens of nosocomial and community-associated infections globally $(2,3)$. Staphylococcus aureus strains carrying the $m e c A$ gene are defined as MRSA. The mecA gene resides on complex mobile genetic elements known as SCCmec ("staphylococcal cassette chromosome" or "staphylococcal chromosomal cassette" containing mecA). Apart from the mecA gene, SCCmec also contains additional genes encoding resistance to other antimicrobials such as aminoglycosides or macrolides (4). Different types of SCCmec have been reported in association with different infections. For example, SCCmec types I, II, and III are generally considered to be MRSA strains associated with healthcare infections, while 
types IV and V are considered to be associated with livestock-associated infections (2,4). MRSA epidemiological studies can also be used in methods such as spa typing to investigate nosocomial and community-acquired infections. Spa typing is based on examining the polymorphism of the gene encoding protein A (spa). Protein A is one of the main virulence factors of $S$. aureus (5). It is also successfully used in multilocus sequence typing (MLST) (sequencing for the interior region of seven different housekeeping genes) to distinguish and monitor bacterial species such as MRSA that cause infectious diseases (6). Aim of our study was to perform in silico analysis of
SCCmec, spa and MLST type, evolutionary similarity and whole genome sequencing (WGS) based antimicrobial susceptibility testing by using genomic data of 30 MRSA strains isolated from human infections in different countries.

\section{MATERIAL AND METHODS}

Whole-genome sequencing (WGS) fasta data of 30 MRSA strains that cause infections in humans and were obtained as etiological agents were included in this study. Genomic data were downloaded from NCBI (www.ncbi.nlm.nih.gov).

Table 1. Descriptive information about MRSA strains included in this study

\begin{tabular}{|c|c|c|c|c|}
\hline & Strain & NCBI Accession Numbers & Host & Country \\
\hline 1 & $\mathrm{HU}-14$ & NZ_LR822061.1/LR822061.1 & Human & Argentina \\
\hline 2 & $\mathrm{BPH} 2003$ & NZ_LR027878.1/LR027878.1 & Human & Australia \\
\hline 3 & BPH2056 & NZ_LR027874.1/LR027874.1 & Human & Australia \\
\hline 4 & BPH2869 & NZ_LR027869.1/LR027869.1 & Human & Australia \\
\hline 5 & $\mathrm{BPH} 2947$ & NZ_LR130515.1/LR130515.1 & Human & Australia \\
\hline 6 & $\mathrm{BPH} 3244$ & NZ_LR027877.1/LR027877.1 & Human & Australia \\
\hline 7 & JKD6004 & NZ_CP040622.1/CP040622.1 & Human & Australia \\
\hline 8 & BLR-DV & NZ_CP058312.1/CP058312.1 & Human & Belarus \\
\hline 9 & Be62 & NZ_CP012013.1/CP012013.1 & Human & Brazil \\
\hline 10 & Bmb9393 & NC_021670.1/CP005288.1 & Human & Brazil \\
\hline 11 & Gv51 & NZ_CP012015.1/CP012015.1 & Human & Brazil \\
\hline 12 & Gv69 & NZ_CP009681.1/CP009681.1 & Human & Brazil \\
\hline 13 & Gv88 & NZ_CP012018.1/CP012018.1 & Human & Brazil \\
\hline 14 & HC1335 & NZ_CP012012.1/CP012012.1 & Human & Brazil \\
\hline 15 & HC1340 & NZ_CP012011.1/CP012011.1 & Human & Brazil \\
\hline 16 & CMRSA-3 & NZ_CP029685.1/CP029685.1 & Human & Canada \\
\hline 17 & CMRSA-6 & NZ_CP027788.1/CP027788.1 & Human & Canada \\
\hline 18 & JK3137 & NZ_CP020960.1/CP020960.1 & Human & Canada \\
\hline 19 & 5_3949 & NZ_LT992462.1/LT992462.1 & Human & Germany \\
\hline 20 & 545 & NZ_CP022908.1/CP022908.1 & Human & Germany \\
\hline 21 & MRSA - AMRF 5 & NZ_CP062467.1/CP062467.1 & Human & India \\
\hline 22 & KG-18 & NZ_AP019543.1/AP019543.1 & Human & Japan \\
\hline 23 & KG-22 & NZ_AP019545.1/AP019545.1 & Human & Japan \\
\hline 24 & TUM9463 & NZ_AP019306.1/AP019306.1 & Human & Japan \\
\hline 25 & aureus & NZ_CP029198.1/CP029198.1 & Human & Korea \\
\hline 26 & NCCP14558 & NZ_CP013953.1/CP013953.1 & Human & Korea \\
\hline 27 & V521 & NZ_CP013957.1/CP013957.1 & Human & Korea \\
\hline 28 & P10 & NZ_CP039157.1/CP039157.1 & Human & Pakistan \\
\hline 29 & NCTC9944 & NZ_LS483309.1/LS483309.1 & Human & UK \\
\hline 30 & FDAARGOS_35 & NZ_CP026072.1/CP026072.1 & Human & USA \\
\hline
\end{tabular}


Table 2. Distribution of similarity, spatype, MLST, SCCmec and WGS based antimicrobial resistance of all MRSA strains included in this study.

\begin{tabular}{|c|c|c|c|c|c|}
\hline Strain & $\begin{array}{c}\text { Similarity } \\
(\%)\end{array}$ & SpaType & MLST & SCCmec & ResFinder \\
\hline HU-14 & 92,64 & t149 & 5 & SCCmec_type_I (1B) & $\begin{array}{c}\text { gyrA, grlA, aac(6')-aph(2"), aph(3')-III, ant(6)- } \\
\text { Ia, ant(9)-Ia, erm(A), mecA }\end{array}$ \\
\hline ВРН2003 & 93,94 & t037 & 239 & $\begin{array}{c}\text { SCCmec_type_III } \\
(3 \mathrm{~A})\end{array}$ & $\begin{array}{c}\text { gyrA, grlA, aph(3')-III, ant(6)-Ia, aac(6')- } \\
\text { aph(2"), ant(9)-Ia, erm(A), dfrG, blaZ, mecA, } \\
\text { tet(K), tet(M) }\end{array}$ \\
\hline ВРH2056 & 92,99 & t037 & 239 & $\begin{array}{c}\text { SCCmec_type_III } \\
(3 \mathrm{~A})\end{array}$ & $\begin{array}{l}\text { aac(6')-aph(2"), ant(6)-Ia, aph(3')-III, ant(9)-Ia, } \\
\text { erm(A), dfrB, blaZ, mecA, tet(K), tet(M) }\end{array}$ \\
\hline ВРH2869 & 93,77 & t037 & 239 & $\begin{array}{c}\text { SCCmec_type_III } \\
(3 \mathrm{~A})\end{array}$ & $\begin{array}{c}\text { gyrA, grlA, qacA, aac(6')-aph(2"), aadD, } \\
\text { ant(9)-Ia, erm(A), dfrB, blaZ, mecA, tet(M) }\end{array}$ \\
\hline ВРН2947 & 93,80 & t037 & 239 & $\begin{array}{c}\text { SCCmec_type_III } \\
(3 \mathrm{~A})\end{array}$ & $\begin{array}{c}\text { gyrA, grlA, grlB, aph(3')-III, aac(6')-aph(2"), } \\
\text { ant(6)-Ia, ant(9)-Ia, ermA, dfrG, blaZ, mecA, } \\
\text { tet(K), tet(M) }\end{array}$ \\
\hline
\end{tabular}

\begin{tabular}{|l|c|c|c|c|c|}
\hline BPH3244 & 93,61 & t1155 & 239 & $\begin{array}{c}\text { SCCmec_type_III } \\
(3 \mathrm{~A})\end{array}$ & $\begin{array}{c}\text { gyrA, grlA, qacA, aac(6')-aph(2"), aadD, } \\
\text { ant(9)-Ia, erm(A), dfrB, tet(M), blaZ, mecA }\end{array}$ \\
\hline JKD6004 & 93,71 & t1959 & 239 & $\begin{array}{c}\text { SCCmec_type_III } \\
(3 \mathrm{~A})\end{array}$ & $\begin{array}{c}\text { grlA, ant(9)-Ia, erm(A), dfrB, tet(M), blaZ, } \\
\text { mecA }\end{array}$ \\
\hline BLR-DV & 93,20 & t037 & 239 & $\begin{array}{c}\text { SCCmec_type_III } \\
(3 \mathrm{~A})\end{array}$ & $\begin{array}{c}\text { grlA, gyrA, ant(6)-Ia, aac(6')-aph(2"), aph(3')- } \\
\text { III, dfrG, blaZ, mecA, rpoB, tet(M), tet(K) }\end{array}$ \\
\hline Be62 & 95,04 & t037 & 239 & $\begin{array}{c}\text { SCCmec_type_III } \\
(3 \mathrm{~A})\end{array}$ & $\begin{array}{c}\text { grlA, gyrA, ant(6)-Ia, aac(6')-aph(2"), aph(3')- } \\
\text { III, ant(9)-Ia, erm(A), dfrB, blaZ, mecA, rpoB, } \\
\text { tet(M) }\end{array}$
\end{tabular}

\begin{tabular}{|l|c|c|c|c|c|}
\hline Bmb9393 & 94,28 & t138 & 239 & $\begin{array}{c}\text { SCCmec_type_III } \\
(3 \mathrm{~A})\end{array}$ & $\begin{array}{c}\text { grlA, gyrA, ant(6)-Ia, ant(9)-Ia, aac(6')- } \\
\text { aph(2"), aph(3')-III, erm(A), dfrG, blaZ, mecA, } \\
\text { rpoB, tet(M) }\end{array}$ \\
\hline Gv51 & 94,63 & t037 & 239 & $\begin{array}{c}\text { SClmec_type_III } \\
(3 \mathrm{~A})\end{array}$ & $\begin{array}{c}\text { grlA, gyrA, ant(6)-Ia, ant(9)-Ia, aac(6')- } \\
\text { aph(2"), aph(3')-III, erm(A), dfrB, blaZ, mecA, } \\
\text { rpoB, tet(M) }\end{array}$
\end{tabular}

\begin{tabular}{|l|c|c|c|c|c|}
\hline Gv69 & 94,99 & t037 & 239 & $\begin{array}{c}\text { SCCmec_type_III } \\
(3 \mathrm{~A})\end{array}$ & $\begin{array}{c}\text { gyrA, ant(6)-Ia, ant(9)-Ia, aac(6')-aph(2"), } \\
\text { aph(3')-III, erm(A), dfrB, blaZ, mecA, rpoB, } \\
\text { tet(M), ileS }\end{array}$ \\
\hline Gv88 & 95,01 & t037 & 239 & $\begin{array}{c}\text { SCCmec_type_III } \\
(3 \mathrm{~A})\end{array}$ & $\begin{array}{c}\text { grlA, gyrA, ant(6)-Ia, ant(9)-Ia, aac(6')- } \\
\text { aph(2"), aph(3')-III, erm(A), dfrB, blaZ, mecA, } \\
\text { rpoB, tet(M) }\end{array}$
\end{tabular}

\begin{tabular}{|l|c|c|c|c|c|}
\hline HC1335 & 94,50 & t138 & 239 & $\begin{array}{c}\text { SCCmec_type_III } \\
(3 \mathrm{~A})\end{array}$ & $\begin{array}{c}\text { grlA, gyrA, ant(6)-Ia, ant(9)-Ia, aph(3')-III, } \\
\text { erm(A), dfrB, blaZ, mecA, rpoB, tet(M), ileS }\end{array}$ \\
\hline
\end{tabular}


Table 2. Continue

\begin{tabular}{|c|c|c|c|c|c|}
\hline HC1340 & 95,08 & t037 & 239 & $\begin{array}{l}\text { SCCmec_type_III } \\
(3 A)\end{array}$ & $\begin{array}{l}\text { grlA, gyrA, ant(6)-la, ant(9)-la, aac(6')-aph(2"), } \\
\text { aph(3')-III, erm(A), dfrB, blaZ, mecA, rpoB, } \\
\text { tet(M), iles }\end{array}$ \\
\hline $\begin{array}{l}\text { CMRSA- } \\
3\end{array}$ & 93,30 & t037 & 241 & $\begin{array}{l}\text { SCCmec_type_III } \\
(3 A)\end{array}$ & $\begin{array}{l}\text { grlA, gyrA, ant(9)-la, erm(A), dfrG, blaZ, mecA, } \\
\text { rpoB, tet(K), tet(M) }\end{array}$ \\
\hline $\begin{array}{l}\text { CMRSA- } \\
6\end{array}$ & 93,96 & t037 & 239 & $\begin{array}{l}\text { SCCmec_type_III } \\
(3 A)\end{array}$ & $\begin{array}{l}\text { grlA, gyrA, ant(6)-la, ant(9)-la, aac(6')-aph(2"), } \\
\text { aph(3')-III, erm(A), dfrG, blaZ, mecA, rpoB, } \\
\text { tet(K), tet(M) }\end{array}$ \\
\hline JK3137 & 96,13 & t008 & 8 & $\begin{array}{l}\text { SCCmec_type_IV } \\
\text { a (2B) }\end{array}$ & grlA, gyrA, mecA \\
\hline 5_3949 & 89,30 & t034 & 398 & $\begin{array}{l}\text { SCCmec_type_Vc } \\
(5 C 2 \& 5)\end{array}$ & dfrG, blaZ, mecA, tet(K), tet(M) \\
\hline 545 & 95,60 & t009 & 254 & $\begin{array}{l}\text { SCCmec_type_IV } \\
\text { (2B) }\end{array}$ & $\begin{array}{l}\text { grlA, gyrA, ant(6)-la, aac(6')-aph(2"), aph(3')- } \\
\text { III, dfrB, mecA, tet(K), tet(M) }\end{array}$ \\
\hline $\begin{array}{l}\text { MRSA - } \\
\text { AMRF } 5\end{array}$ & 90,80 & t657 & 772 & $\begin{array}{l}\text { SCCmec_type_V } \\
(5 \mathrm{C} 2)\end{array}$ & $\begin{array}{l}\text { grlA, gyrA, ant(6)-la, aac(6')-aph(2"), aph(3')- } \\
\text { III, erm(A), msr(A), mph(C), dfrG, blaZ, mecA }\end{array}$ \\
\hline KG-18 & 92,58 & t17639 & 5 & $\begin{array}{l}\text { SCCmec_type_II } \\
(2 A)\end{array}$ & $\begin{array}{l}\text { gyrA, grlA, grlB, aaD, aac(6')-aph(2"), ant(9)- } \\
\text { la, erm(A), mecA, tet(M) }\end{array}$ \\
\hline KG-22 & 92,92 & t17639 & 5 & $\begin{array}{l}\text { SCCmec_type_II } \\
(2 A)\end{array}$ & $\begin{array}{l}\text { gyrA, grlA, grlB, aaD, aac(6')-aph(2"), ant(9)- } \\
\text { la, erm(A), mecA, tet(M) }\end{array}$ \\
\hline $\begin{array}{l}\text { TUM946 } \\
3\end{array}$ & 94,54 & t539 & 2389 & $\begin{array}{l}\text { SCCmec_type_II } \\
(2 A)\end{array}$ & $\begin{array}{l}\text { gyrA, } \operatorname{grl} A, \operatorname{aac}\left(6^{\prime}\right)-\operatorname{aph}\left(2^{\prime \prime}\right), \operatorname{ant}(9)-\operatorname{la}, \operatorname{erm}(A) \text {, } \\
\text { dfrB, mecA, tet(M), }\end{array}$ \\
\hline aureus & 94,10 & t002 & 5 & $\begin{array}{l}\text { SCCmec_type_II } \\
(2 A)\end{array}$ & $\begin{array}{l}\text { gyrA, grlA, aac(6')-aph(2"), ant(9)-la, erm(A), } \\
\text { rpoB, mecA, tet(M), fusC }\end{array}$ \\
\hline $\begin{array}{l}\text { NCCP14 } \\
558\end{array}$ & 94,20 & t045 & 5 & $\begin{array}{l}\text { SCCmec_type_II } \\
(2 A)\end{array}$ & $\begin{array}{l}\text { gyrA, grlA, aadD, ant(9)-la, erm(A), mecA, } \\
\text { tet(M) }\end{array}$ \\
\hline V521 & 94,64 & t037 & 239 & $\begin{array}{l}\text { SCCmec_type_III( } \\
\text { 3A) }\end{array}$ & $\begin{array}{l}\text { gyrA, grlA, aph(3')-III, ant(6)-la, aac(6')- } \\
\text { aph(2"), ant(9)-la, erm(A), dfrG, tet(K), tet(M), } \\
\text { blaZ, mecA }\end{array}$ \\
\hline P10 & 94,68 & t064 & 113 & $\begin{array}{l}\text { SCCmec_type_IV } \\
\text { c (2B) }\end{array}$ & $\begin{array}{l}\text { grlA, gyrA, ant(6)-la, ant(9)-la, aac(6')-aph(2"), } \\
\text { aph(3')-III, dfrG, blaZ, mecA, rpoB, tet(K), fusA, } \\
\text { mupA }\end{array}$ \\
\hline $\begin{array}{l}\text { NCTC99 } \\
44\end{array}$ & 93,14 & t037 & 240 & $\begin{array}{l}\text { SCCmec_type_III } \\
(3 A)\end{array}$ & $\begin{array}{l}\text { aac(6')-aph(2"), ant(9)-la, erm(A), dfrG, blaZ, } \\
\text { mecA, tet(K), tet(M) }\end{array}$ \\
\hline $\begin{array}{l}\text { FDAARG } \\
\text { OS_35 }\end{array}$ & 100,00 & t064 & 507 & $\begin{array}{l}\text { SCCmec_type_IV } \\
\text { d (2B) }\end{array}$ & gyrA, grlA, aadD, blaZ, mecA, tet(M) \\
\hline
\end{tabular}


Descriptive information about all these MRSA strains were shown in Table 1.

All in silico analysis with fasta files were performed on Center for Genomic Epidemiology (CGE) website (https://www.genomicepidemiology.org/) with related softwares. Phylogeny, alignment or similarity analysis with large data were performed via CSI Phylogeny online software (7). SCCmec, spa and MLST typing were performed using with softwares at Center for Genomic Epidemiology (CGE) website $(8,9,10)$. ResFinder 4.0 softwares were used to performe whole genome sequencing (WGS) based antimicrobial susceptibility testing (AST) (11).

\section{RESULTS}

After phylogeny analysis, according to FDAARGOS_35 strain from USA, similarity percentage of strains were shown in Table 2. The evolutionary tree of these strains were shown in Figure 1. Strain 5_3949 from Germany was the most distant similarity to FDAARGOS 35 strain and similarity was found $89.3 \%$. FDAARGOS_35 strain
Canada was found the most similar strain to FDAARGOS 35 and similarity was found $96.13 \%$. Strain JK3137 was also found SCCmec type_IVa(2B). Strains 545 from Germany and $\mathrm{HC} 1340$ from Brazil similarities were found $95.60 \%$ and $95.08 \%$ respectively. When the spa types were analysed, 14 different spa types were detected of all MRSA strains. Spa type 037 was most detected. $14(46.6 \%)$ out of 30 MRSA strains were found spa type t037. Spa type t064, t138 and t17639 were found 2 MRSA strains. 10 MRSA strains showed unique spa types. (Table 2). When the MLST were analysed, 11 different sequence types were found via MLST. ST239 was most detected 16 (53.3\%) out of 30 MRSA strains. ST5 was found the second one at 5 out of 30 strains. The other 9 nine ST were found unique strain (Table 2). When the SCCmec type were analysed, 9 different SCCmec types were found. $18(60 \%)$ out of 30 MRSA strains were found SCCmec_type_III(3A). 5 MRSA strains were found SCCmec_type_II(2A). 7 strains showed unique SCCmec types. When the WGS based

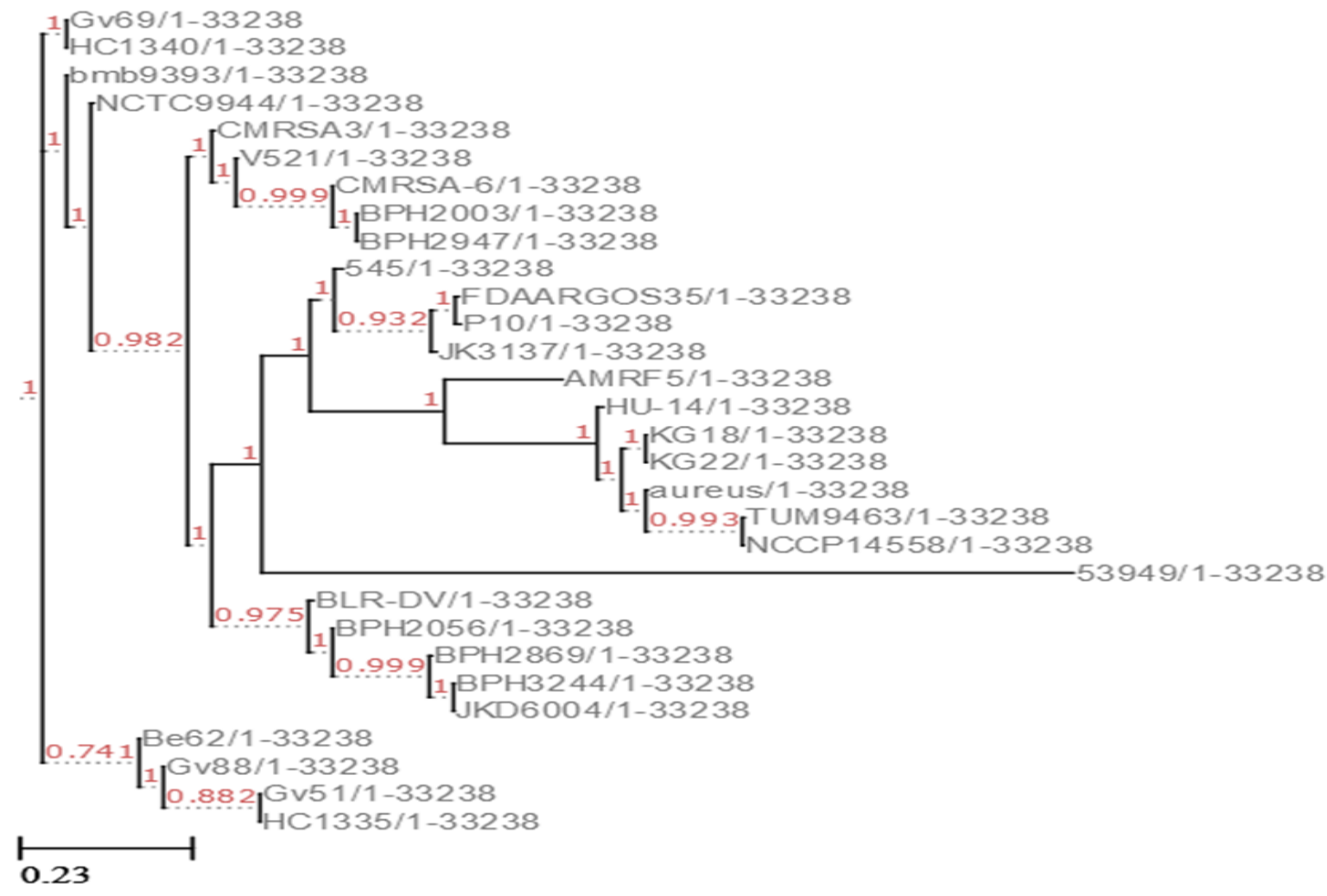

Figure 1. Maximum-likelihood trees for large alignments of MRSA strains.

was found SCCmec_type_IVd(2B), on the other hand, Strain 5_3949 was found SCCmec_type_Vc(5C2\&5). Strain JK3137 from antimicrobial susceptibility testing results were analysed, 28 out of 30 MRSA strains were carrying aminoglycoside resistance gene such as aac(6')- 
aph(2"), aph(3')-III and ant(6)-la. 27 strains were carrying tetracycline resistance genes such as tet(K) or tet(M). 26 strains were carrying fluoroquinolone resistance genes and single nucleotide polymorphisms (SNPs) such as gyrA (p.S84L) or grlA (p.S80F). 24 out of 30 strains were carrying macrolide and folate pathway antagonist resistance genes such as $\operatorname{erm}(\mathrm{A})$ or $\mathrm{dfrB}$ respectively (Table 2 ).

\section{DISCUSSION}

The incidence of MRSA is still increasing and remains an important public health problem $(1,12)$. The increase in resistant strains creates serious difficulties in the treatment. In order to develop new drugs, it is important to detect virulence and resistance genes in multi-resistant strains such as MRSA. In recent years, big data obtained with WGS technologies have provided tremendous developments. It can be used for typing strains such as MRSA, detecting antibiotic resistance and virulence genes, and examining their genomic materials (12).

Zhou et al, in their study, reported that when they examined the phylogenetic tree of different MRSA strains, they thought that the strains from different geographies were similar to each other and that the strains could migrate frequently between geographic regions (13). We found a similar result in the strains we examined in our study.

Asadollahi et al. reported spa types detected in different geographical regions in their study. It has been reported that 037 is the most detected spa type in Asia and Africa (14). Neela et al. also reported in their study that $83 \%$ of the strains in Malaysia were spa type t037 (15). Stańkowska et al. reported that they detected spa type t037 with high prevalence in Poland (16). These data showed us that as Zhou et al (13) stated, the strains migrated seriously and these strains could be encountered in different geographical regions.

In our study, ST239 was most detected 16 (53.3\%) out of 30 MRSA strains. Neela et al. also reported in their study that $83 \%$ of the strains in Malaysia were ST239 (15). Asadollahi et al. reported some spa types constantly associated with some STs such as ST5 or ST239 (14). Dai et al., in their study in China, reported that the prevalence of ST239 and t037 types was decreased in MRSA isolates, whereas ST5 and t2460 types were seen in isolates. They also stated that there was an increase in the prevalence of ST398 type. They said that they did not know exactly what the reason was (17). Although not all of our strains originate from Asia, we found that the frequency of ST239 and ST5 was high after our in silico analysis. Gostev and Siderenko reported in their study that SCCmec I, II and III were detected together with hospital-acquired epidemic strains such as ST5 and ST8, and SCCmec IV began to be detected in different geographical locations (18). Neela et al. also reported in their study that ST5-SCCmec type II and ST239-SCCmec type III appeared mostly together (15). We detected SCCmec_type_III(3A) in our study after our in silico analysis. Although we included strains from different countries in the in silico analysis, we obtained results similar to those reported by Neela et al. (15).

Mohammadi et al. reported that SCCmec type III strains contained aminoglycoside resistance genes in MRSA isolates they examined in their study. They also reported that they contain especially the ermA gene for erythromycin (19). Ebrahim-Saraie et al. reported that the resistance rates of SCCmec type III MRSA isolates they examined in their study were lower than the resistance rate of SCCmec type I and II strains, but they had serious antimicrobial resistance (20). In our study, we observed that MRSA strains carry genes associated with severe antimicrobial resistance after wgs-based antimicrobial resistance testing, and that although there may be slight differences in different geographical locations, the rates of resistance to major antimicrobial classes may be very high.

\section{CONCLUSION}

As a conclusion, after our in silico analysis results, we found that similar typing profiles could be observed in the strains in different geographical locations. we realized that certain types of spa, MLST and SCCmec can coexist. We believe that these types and their antimicrobial resistance profiles should be continuously followed up with these new techniques.

Author contribution: Concept: M.D., A.Y., S.E, Design: M.D., Analysis or Interpretation: M.D., A.Y., S.E, Literature Search: M.D., A.Y., S.E, Writing: M.D., A.Y., S.E.

Conflict of interests: The authors declare that there is no conflict of interest in the content of the article.

Peer-review: Externally peer-reviewed.

\section{REFERENCES}

1. Kulkarni A. P., Nagvekar V. C., Veeraraghavan B., Warier A. R., Ahdal J., Jain R.: Current Perspectives on Treatment of Gram-Positive 
Infections in India: What Is the Way Forward? Interdiscip Perspect Infect Dis 2019; 2019: 7601847.

2. Sarkhoo E., Udo E. E., Boswihi S. S., Monecke S., Mueller E., Ehricht R. The Dissemination and Molecular Characterization of Clonal Complex $361 \quad$ (CC361) Methicillin-Resistant Staphylococcus aureus (MRSA) in Kuwait Hospitals. Front Microbiol 2021; 12: 658772.

3. Shang L., Li J., Song C., et al. Hybrid Antimicrobial Peptide Targeting Staphylococcus aureus and Displaying Anti-infective Activity in a Murine Model. Front Microbiol. 2020; 11: 1767.

4. Monecke S, Coombs G, Shore AC, et al. A field guide to pandemic, epidemic and sporadic clones of methicillin-resistant Staphylococcus aureus. PLoS One 2011; 6(4): e17936.

5. Mohammed KAS, Abdulkareem ZH, Alzaalan AR, Yaqoob AK. Spa typing of Staphylococcus aureus Isolated from Clinical Specimens from Outpatients in Iraq. Pol J Microbiol 2021; 70(1): 79-85.

6. Liu Y, Ji Y. Multilocus Sequence Typing of Staphylococcus aureus. Methods Mol Biol. 2020; 2069: 95-102.

7. Kaas RS, Leekitcharoenphon P, Aarestrup FM, Lund $O$. Solving the problem of comparing whole bacterial genomes across different sequencing platforms. PLoS One 2014; 9(8): e104984.

8. International Working Group on the Classification of Staphylococcal Cassette Chromosome Elements (IWG-SCC). Classification of staphylococcal cassette chromosome mec (SCCmec): guidelines for reporting novel $\mathrm{SCCmec}$ elements. Antimicrob Agents Chemother 2009; 53(12): 4961-7.

9. Bartels $M D$, Petersen $A$, Worning $P$, et al. Comparing whole-genome sequencing with Sanger sequencing for spa typing of methicillinresistant Staphylococcus aureus. J Clin Microbiol. 2014; 52(12): 4305-4308.

10. Larsen MV, Cosentino S, Rasmussen S, Friis C, Hasman H, Marvig RL, Jelsbak L, SicheritzPontén T, Ussery DW, Aarestrup FM, Lund O. Multilocus sequence typing of total-genomesequenced bacteria. J Clin Microbiol 2012; 50(4): 1355-61.

11. Bortolaia V, Kaas RS, Ruppe E, Roberts MC, Schwarz S, Cattoir V, Philippon A, Allesoe RL, Rebelo AR, Florensa AF, Fagelhauer L, Chakraborty T, Neumann B, Werner G, Bender
JK, Stingl K, Nguyen M, Coppens J, Xavier BB, Malhotra-Kumar S, Westh H, Pinholt M, Anjum MF, Duggett NA, Kempf I, Nykäsenoja S, Olkkola $S$, Wieczorek K, Amaro A, Clemente L, Mossong J, Losch S, Ragimbeau C, Lund O, Aarestrup FM. ResFinder 4.0 for predictions of phenotypes from genotypes. J Antimicrob Chemother 2020; 75(12): 3491-3500.

12. Ali MS, Isa NM, Abedelrhman FM, Alyas TB, Mohammed SE, Ahmed AE, Ahmed ZSA, Lau NS, Garbi MI, Amirul AA, Seed AO, Omer RA, Mohamed SB. Genomic analysis of methicillinresistant Staphylococcus aureus strain SO-1977 from Sudan. BMC Microbiol. 2019 Jun 11; 19(1): 126.

13. Zhou W, Li X, Osmundson T, Shi L, Ren J, Yan $H$. WGS analysis of ST9-MRSA-XII isolates from live pigs in China provides insights into transmission among porcine, human and bovine hosts. J Antimicrob Chemother 2018 Oct 1; 73(10): 2652-2661.

14. Asadollahi $P$, Farahani NN, Mirzaii $M$, Khoramrooz SS, van Belkum A, Asadollahi K, Dadashi M, Darban-Sarokhalil D. Distribution of the Most Prevalent Spa Types among Clinical Isolates of Methicillin-Resistant and -Susceptible Staphylococcus aureus around the World: A Review. Front Microbiol 2018; 9: 163.

15. Neela V, Ghasemzadeh Moghaddam $H$, van Belkum A, Horst-Kreft D, Mariana NS, Ghaznavi Rad E. First report on methicillin-resistant Staphylococcus aureus of Spa type T037, Sequence Type 239, SCCmec type III/IIIA in Malaysia. Eur J Clin Microbiol Infect Dis 2010; 29(1): 115-7.

16. Stańkowska M, Garbacz K, Piechowicz L, Bronk M. Dissemination Of t437-SCCmecIV And Coagulase-Negative t037-SCCmeclll Types Among Borderline Oxacillin-Resistant Staphylococcus aureus Isolated From Skin Infections And Diabetic Foot Ulcers. Infect Drug Resist 2019; 12: 3197-3203.

17. Dai Y, Liu J, Guo W, et al. Decreasing methicillinresistant Staphylococcus aureus (MRSA) infections is attributable to the disappearance of predominant MRSA ST239 clones, Shanghai, 2008-2017. Emerg Microbes Infect 2019; 8(1): 471-478.

18. Gostev VV, Sidorenko SV. [Staphylococcal cassette chromosome mec, evolution and genetic lines of methicillin resistant 
Staphylococcus aureus]. Antibiot Khimioter. 2012; 57(9-10): 38-46.

19. Mohammadi S, Sekawi Z, Monjezi A, Maleki MH, Soroush S, Sadeghifard N, Pakzad I, AziziJalilian F, Emaneini M, Asadollahi K, Pourahmad $F$, Zarrilli $R$, Taherikalani M. Emergence of SCCmec type III with variable antimicrobial resistance profiles and spa types among methicillin-resistant Staphylococcus aureus isolated from healthcare- and communityacquired infections in the west of Iran. Int J Infect Dis 2014; 25: 152-8.

20. Ebrahim-Saraie HS, Motamedifar M, Sarvari J, Hoseini Alfatemi SM. Emergence of SCCmec Type I Obtained From Clinical Samples in Shiraz Teaching Hospitals, South-West of Iran. Jundishapur J Microbiol 2015; 8(6): e16998. 